\title{
Novel topical fluoride-delivery system for remineralization of dental enamel: optimization studies
}

\author{
P.J. Spooner, Y. Kanaya, J.L. Fox and W.I. Higuchi \\ College of Pharmacy. The University of Michigan, Ann Arbor, MI 48109 (U.S.A.)
}

(Received August 2nd, 1982)

(Accepted November 18th, 1982)

\section{Summary}

Further studies have been conducted on a calcium fluoride delivery system with a view to optimizing its ability to remineralize dental enamel. The influence of particle size and load of calcium fluoride used, as well as treatment time, was evaluated in vitro on prior demineralized bovine enamel. Initial studies with the original singlemembrane system did not allow an unambiguous interpretation of efficacy, due to persistent interference from calcium fluoride particles. To overcome this problem. the system was modified to include a second membrane as protection for the enamel surface. This double-membrane system revealed a marked particle size and load effect on fluoride delivery. The performance of a high load of small size calcium fluoride particles was significantly better than previous results obtained with the system and approached the most effective remineralizing conditions established with solution fluoride.

\section{Introduction}

Work in our laboratories has led to the development of a prototype fluoride-containing delivery system for the remineralization of prior-demineralized dental enamel (Yonese et al., 1981b) which may be capable of remineralizing natural caries lesions in vivo by deposition of fluorapatite (FAP), a more acid-resistant mineral than hydroxyapatite, the major component of dental enamel.

The strategy for development of the therapeutic system was based on fundamental studies performed in these laboratories with remineralizing solutions (Yonese et al., 1981a). These showed that remineralization of artificial lesions in bovine enamel 
can be very successful when the ion activity product $\mathrm{K}_{\mathrm{FAP}}\left(\mathrm{a}_{\mathrm{Ca}^{2}}^{10} \times \mathrm{a}_{\mathrm{PO}_{4}^{2-}}^{6} \times \mathrm{a}_{\mathrm{F}}^{2}\right)$ is $\sim 10^{-108}$. This determined the choice of calcium fluoride for the prototype system since this solute should provide sustained $F$ release at levels prescribed by the best remineralizing solution conditions.

Yonese et al. (1981b) and Fox et al. (1981) described the in vitro testing of the device on demineralized bovine and human enamel, in which a nominal loading of micronized calcium fluoride was retained at the lesion surface by covering with a semi-permeable membrane. The fluoride uptake observed in these studies was reasonably promising but significantly less than the best results obtained with solution fluoride. Preliminary in vivo testing (Abrahams et al., 1980) on demineralized enamel blocks affixed in the oral cavity, also gave low fluoride levels compared with the optimum solution treatment.

This less than optimum performance of the delivery system necessitated a systematic evaluation of how certain features of the system design influenced the remineralizing efficacy. In this study the importance of particle size and load of calcium fluoride used was assessed, for various times of remineralization. It was hoped that this information could lead to an improved system which would justify an extensive evaluation of efficacy in vivo.

\section{Materials and methods}

To ensure good reproducibility in the results, the techniques employed were very similar to the standard in vitro procedures established in the previous work (Yonese et al.. 198 (b).

Bovine teeth from 8-week-old, crate-fed calves were ground to remove their pellicle as described previously (Yonese et al., 1981b). A $0.25 \mathrm{~cm}^{2}$ window was ascribed on the labial surface of each tooth with dental inlay wax. The exposed enamel was then dernineralized by agitating in $0.1 \mathrm{M}$ acetate buffer at $\mathrm{pH} 4.5$. containing $3 \mathrm{mM}$ calcium and phosphate and sufficient sodium chloride for an ionic strength of 0.5 .

The particle size range for calcium fluoride samples was estimated by viewing specimens, dispersed in saturated calcium fluoride solution with oil-immersion light microscopy at $1000 \times$ magnification. A graduated eyepiece permitted particles greater than $1 \mu \mathrm{m}$ to be measured.

Sub-micron size calcium fluoride particles were prepared by spontaneous nucleation from mixing solutions of $1.25 \times 10^{-2} \mathrm{M}$ calcium chloride and $2.5 \times 10^{-2} \mathrm{M}$ sodium fluoride in equal volumes. Radiolabeled calcium fluoride was prepared for some experiments by introducing ${ }^{45} \mathrm{Ca}$ into the calcium chloride solution. The dispersed solid was collected and rinsed well to remove labile ions.

Large size calcium fluoride crystals were isolated from a ground and sieved commercial sample ${ }^{1}$ by sedimentation in saturated calcium fluoride solution. A $5 \mathrm{~g}$

Optical Grade Crystals, Ventron, Beverly. MA, U.S.A. 
quantity of the sample was dispersed in $200 \mathrm{ml}$ of the solution and allowed to settle for $30 \mathrm{~s}$ before decanting and discarding the suspension above the sediment. After repeating this procedure 5 times, the supernatant was essentially free from particles

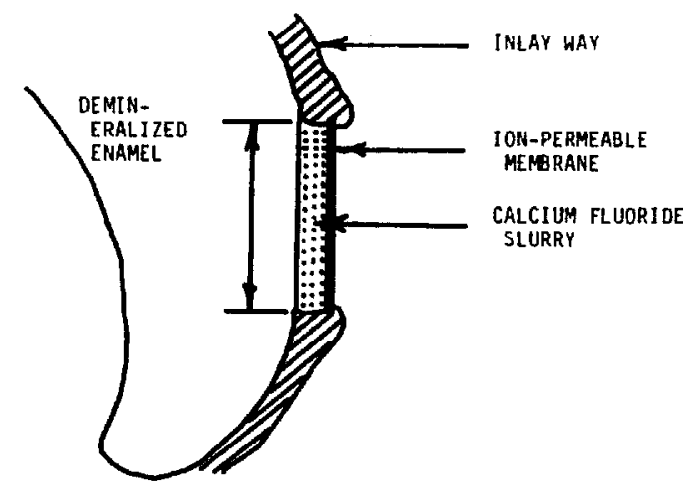

SINGLE-MERBRANE SYSTEM

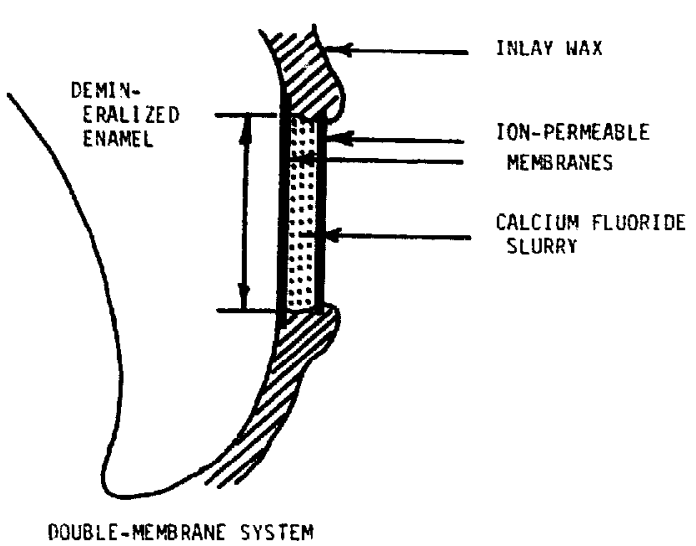

Fig. 1. Schematic diagram of the calcium fluoride delivery systems.

after the $30 \mathrm{~s}$ sedimentation period. The sedimented particles were mostly $15-30 \mu \mathrm{m}$ in size.

\section{System design and application}

The configuration of the deliverv devices used is illustrated schematically in Fig. 1. These were prepared to con ? $\cdot$ a 0.5 or $5 \mathrm{mg}$ loading of the calcium flucride. The single-membrane system was e sentially the same as the prototype system (Type B) used in the previous study (Yonese et al., 1981a). Here, a slurry of the calcium fluoride was applied directly to the demineralized enamel surface and then covered with an ion-permeable membrane ${ }^{2}$. The double-membrane system differs only in that it also has an ion-permeable membrane covering the demineralized enamel surface to guard against contamination from the calcium fluoride.

${ }_{2}^{2}$ Spectrapor Dialysis Membrane (6000-8000 inolecular weight cutoff). 
The remineralizing treatment involved agitating the samples in a $0.1 \mathrm{M}$ acelate buffer solution at $\mathrm{pH} 4.5$, containing $12 \mathrm{mM}$ calcium and phosphate and sufficient sodium chloride for an ionic strength of 0.5 . Treatments were conducted at $30^{\circ} \mathrm{C}$ for 24 . 48 or $96 \mathrm{~h}$ with periodic changing of the remineralizing solution to prevent significant accumulation of solution fluoride.

\section{Quantitative assessment of remineralization}

Enamel surfaces treated with the single membrane system were rinsed thoroughly with double-distilled water and then abraded with a foam pellet coated with carborundum paste to remove residual calcium fluoride. The abraded surfaces weie then biopsied by immersion in $0.5 \mathrm{~N}$ perchloric acid for successive intervals of 30 , $60,60,120$ and $200 \mathrm{~s}$. The abrasion samples were extracted with $0.5 \mathrm{~N}$ perchloric acid and analyzed along with the biopsied samples for phosphate and fluor de. Phosphorus was assayed by the method of Gee et al. (1954), in which the phosphoammonium-molybdate complex was reduced with stannous chloride and absorbance of the resulting blue color measured at $720 \mathrm{~nm}$. Fluoride was determined with a fluoride-sensitive combination electrode ${ }^{3}$ after mixing the samples with suitable quantities of a Total Ionic Strength Adjusting Buffer (TISAB).

The enamel surfaces treated with the double-membrane system were biopsied directly by the acid-etch procedure described above and also analyzed for phosphate and fluoride.

\section{Results and discussion}

Single-membrane system

The fluoride uptake measured from the single-membrane device is shown as a function of enamel depth in Fig. 2, for remineralization times of 24,48 and $96 \mathrm{~h}$, respectively. Enamel depihs are estimates based on the amount of phosphate removed in the biopsy and assuming that the treated enamel has the density of pure

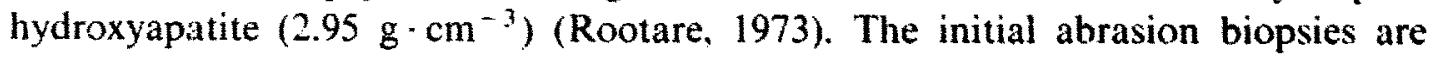
omitted from the plots since these were most likely to include interference from residual calcium fluoride. The results show a large dependence in fluoride uptake on loading and treatment time for small size calcium fluoride particles. On the other hand, the low uptake from the larje size crystal showed little improvement beyond $24 \mathrm{~h}$ at either loading.

The far superior fluoride uptake from the high loading of sub-micron calcium fluoride particles was appreciably greater than that achieved in previous studies with this device (Yonese et al., 1981b). However, the results are obviously suspect since these fluoride levels ( $10,000 \mathrm{ppm}$ up to $50 \mu \mathrm{m}$ depths) are also much higher than could be expected from fluorapatite deposition alone. The most favorable conditions for fluorapatite deposition provides no more than about $2000-4000 \mathrm{ppm}$ fluoride at around $30-50 \mu \mathrm{m}$ depths, as shown from the solution treatment in Fig. 4. It must

\footnotetext{
Orion Research.
} 

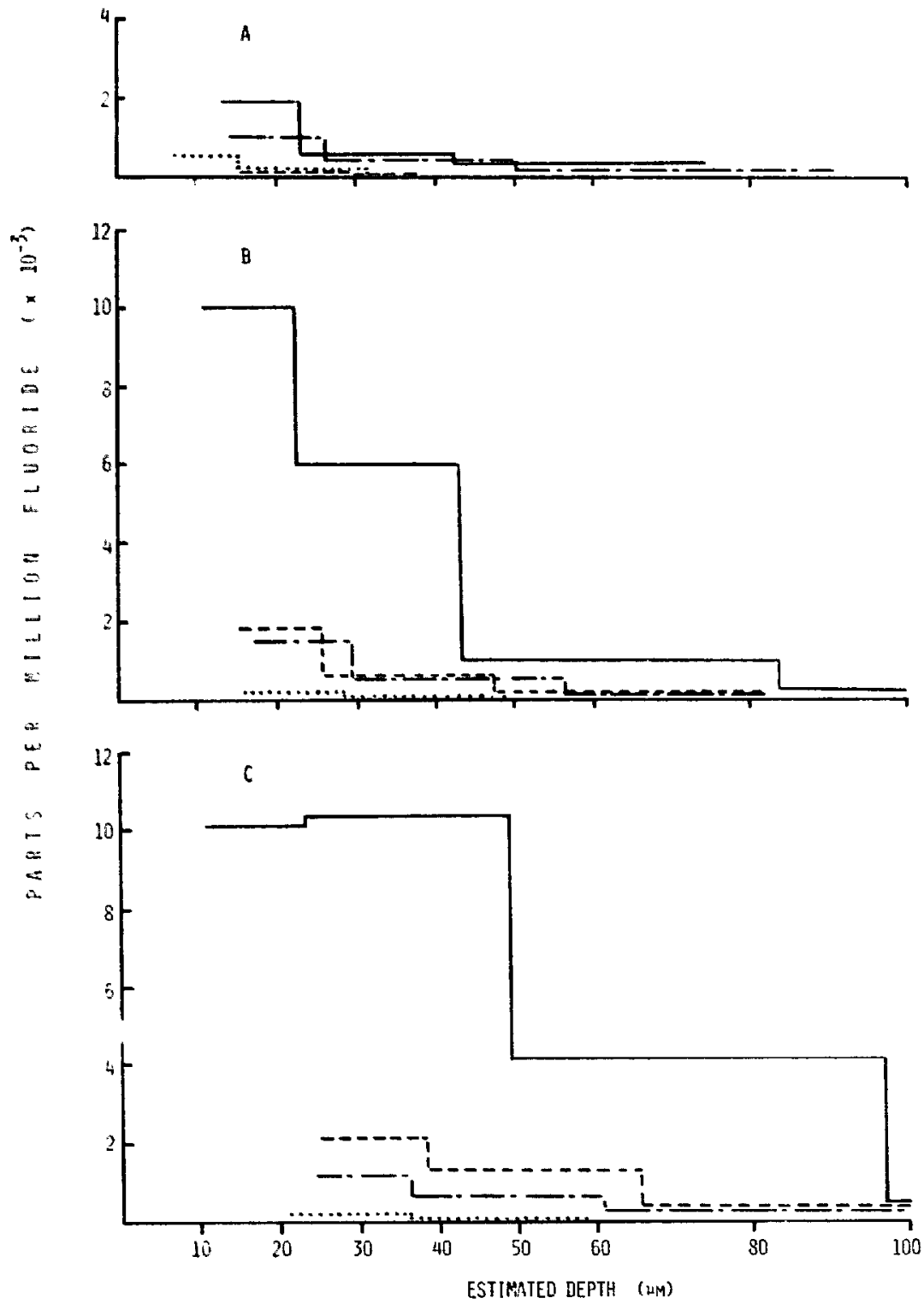

Fig. 2. Single-membrane system performance: fluoride uptake after $24 \mathrm{~h}$ (A), $48 \mathrm{~h}$ (B), and $96 \mathrm{~h} \mathrm{(C)}$ of treatment. Profiles are for sub-micron calcium fluoride at $5 \mathrm{mg} \mathrm{(-}-$ ) and $0.5 \mathrm{mg} \mathrm{(- \ldots .)}$ loat, and large size calcium nuoride particles at $5 \mathrm{mg}(-\ldots)$ and $0.5 \mathrm{mg}(\cdots))$ load.

thercfore be assumed that significant amounts of unreacted calcium fluoride are also taken up during treatment with the single-membrane device. Verification of this was given by the appearance of large amounts of ${ }^{45} \mathrm{Ca}$ in the surface layer of enamel, after treating with ${ }^{45} \mathrm{CaF}_{2}$ in the delivery system. This should represent only unreacted calcium fluoride since very little ${ }^{45} \mathrm{Ca}$ will be available for remineralization compared with 'cold' calcium from the remineralizing solution.

The interference from calcium fluoride appears to pose serious problems in interpreting the extent of remineralization from the fluoride uptake, even after 

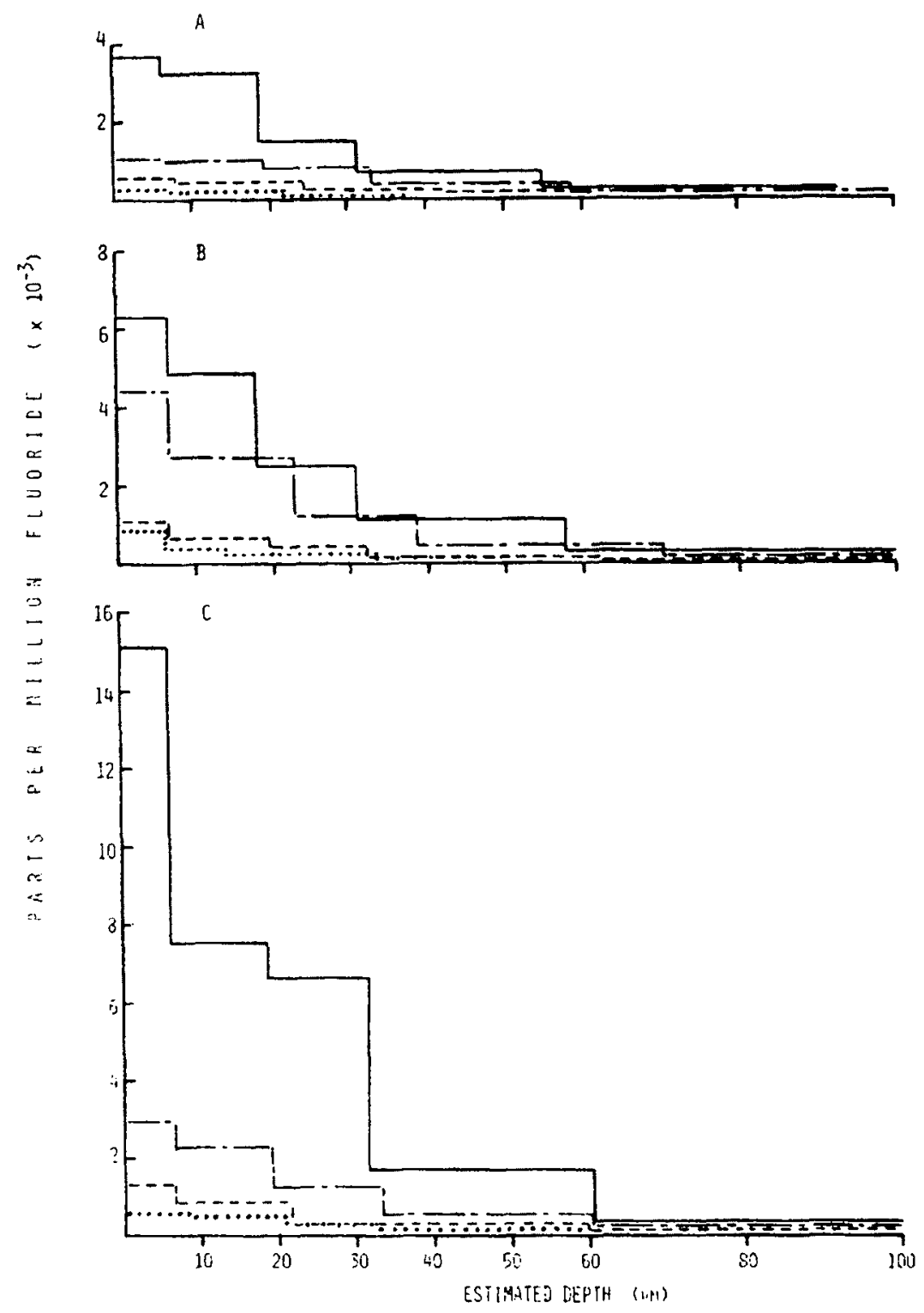

Fig. 3. Double-membrane system performance: fluoride uptake after $24 \mathrm{~h} \mathrm{(A).} 48 \mathrm{~h}$ (B). and $96 \mathrm{~h}$ (C). of treatment. Profiles are for sub-micron calcium fluoride at $5 \mathrm{mg}(-\ldots)$ and $0.5 \mathrm{mg}(\ldots \ldots)$ load, and large size calcium fluoride particles at $5 \mathrm{mg}(-\ldots .+)$ and $0.5 \mathrm{mg}(\cdots$,$) load.$

removing a layer from the enamel surface by abrasion. It is possible that the calcium fluoride interference was associated in some way with the remineralization process since it became more apparent under conditions likely to be most favorable for remineralization (high loading of small 'e calcium fluoride). An obvious explanation of this is that calcium fluoride particles become entrapped during deposition of fluoridated-apatite. If this is true then the above results will have some qualitative value by exhibiting the rank order in remineralizing effectiveness. 


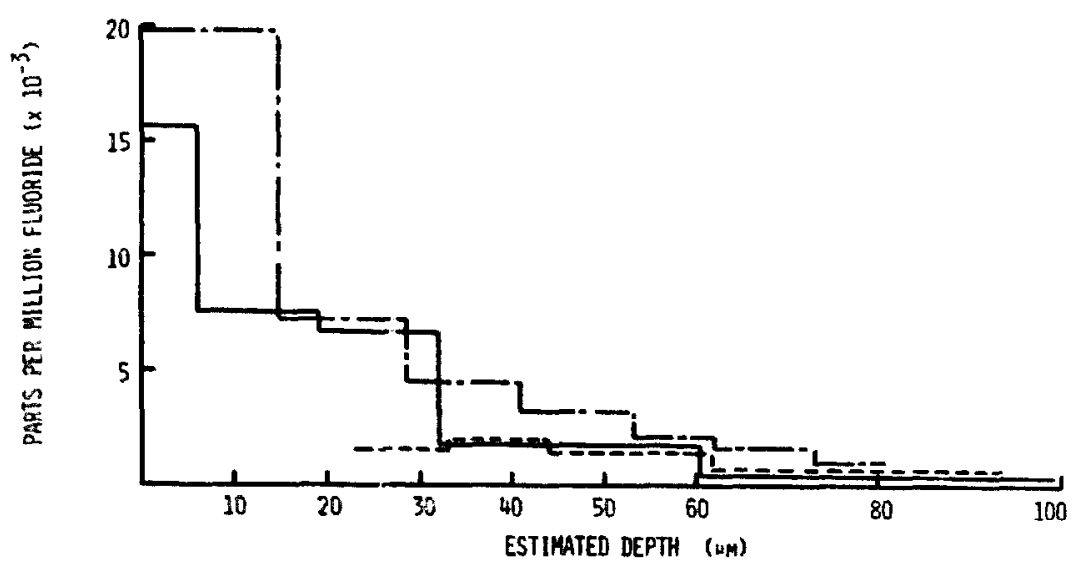

Fig. 4. Fluoride uptake from the best solution remineralization (-_-_-) and from $5 \mathrm{mg}$ load of sub-micron calcium đuoride after $96 \mathrm{~h}$ in the double-membrane (_- ) and single (- . . . -) membrane systems. The latter was corrected for calcium fluoride interference.

\section{Double-membrane system}

To obtain a more quantitative assessment of remineralizing effectiveness, the foregoing series of conditions were reproduced using the double-membrane system. The enamel in these experiments was not exposed directly to the calcium fluoride and so the possibility of serious interference from unreacted calcium fluoride was eliminated. The fluoride uptake measured under these conditions is presented in Fig. 3 for the 3 treatmen times. From our experience of treatment with remineralizing solutions and considering the precautions taken against contamination with calcium fluoride, we can reasonably expect these levels to represent apatitic fluoride uptake.

The small size calcium fluoride particles showed a large dependence on loading. which increased with duration of treatment. The high load of small size calcium fluoride produced significantly better remineralization than the high load of large size crystals, especially beyond the $48 \mathrm{~h}$ treatment. The smaller loadings of both calcium fluoride samples produce low remineralization with little improvement beyond the $24 \mathrm{~h}$ treatment. Overall, the results show a significant improvement in performance for the smaller particle size and increased loading of calcium fluoride. Treatment time became a significant factor when using a favorable combination of particle size and load. These trends are approximated in the results from the single membrane system, and so, as suggested earlier, the interference from calcium fluoride from this system may indeed have been a consequence, at least in part, of the remineralization process.

The best remineralizing performance for the device approaches that achieved under the most favorable solution conditions, as shown in Fig. 4, but only after a prolonged period of treatment. Also presented are predicted apatite-fluoride levels from the single membrane system with the same conditions of high load, sub-micron calcium fluoride after $96 \mathrm{~h}$ treatment. These results were obtained by using radiolabeled calcium fluoride and making the maximum possible correction for contamination by assuming all ${ }^{45} \mathrm{Ca}$ in enamel as being unreacted calcium fluoride. The resulting fluoride levels at around $30-50 \mu \mathrm{m}$ are essentially the same as those given 
by the double-membrane system. This similarity between the performance of the single- and double-membrane systems is encouraging since it supports the concept proposed previously (Yonese et al., 1981b) that remineralization can be controlled merely by the dissolution characteristics of the calcium fluoride, thus permitting greater flexibility for system design.

\section{Conclusions}

The best remineralizing performance observed here represents a significant improvement over previous results with the delivery system and is an important advance in terms of system optimization. With this knowledge, the system can now be more confidently applied under conditions closer to the therapeutic situation.

There are a number of possible explanations for the observed effect of particle size and load of calcium fluoride on the fluoride availability from the device. The most likely of these are: (1) differences in intrinsic solubility-departure from normal thermodynamic solubility behavior with sub-micron calcium fluoride, due to the extreme smallness of the particles (Gibbs-Kelvin effect) or polymorphic variations in this sample; (2) a simple surface area effect on the rate of dissolution or solution reaction rate; and (3) an indirect surface area effect on the rate of dissolution resulting from a surface 'poisoning' by components of the remineralizing medium.

The importance of these phenomena in relation to the effects observed here is being systematically investigated in these laboratories. This information may well provide further opportunities for improving the effectiveness of the delivery system.

\section{Acknowledgement}

Supported by NIDR Grants DE0273I and DE04600.

\section{References}

Abrahams, L.J., Yonese, M., Higuchi, W.I., Fox, J.L. and Charbeneau G.T., In vivo remineralization using a sustained wpical fluoride delivery system. J. Dent. Res., 59 (1980) 583-587.

Fox, J.L.. Yonese. M., Iyer, B.V., Abrahams, L.J. and Higuchi, W.I. Novel topical fluoride delivery system II: Remineralization of human teeth. J. Pharm. Sci., 70 (1981) 910-912.

Gee. A., Domi ngues. L. and Deitz, V., Determination of inorganic constituents in sugar. Anal. Chem.. 26 (1954) $1487-1492$.

Rootare. H.M. Free surface energies, heats of solution and heats of immersion of calcium apatite powders, Ph.D. Thesis, The University of Michigan, 1973.

Yonese, M. Fox, J.L.. Nambu, N., Hefferren, J.J. and Higuchi, W.I., Fluoride remineralization of demineralized bovine tooth enamel and hydroxyapatite pellets. J. Pharm. Sci., 70 (1981a) 904-907.

Yonese, M. Iyer, B.Y., Fox, J.L.. Hefferren, J.J. and Higuchi, W.I., Novel topical nuoride delivery system 1: Remineralization of ground bovine teeth. J. Pharm. Sci., 70 (1981b) 907-910. 